\title{
食物中 $\mathrm{Ni}^{2+}$ 胁迫对斜纹夜蛾幼虫中肠细胞解毒酶的影响
}

\author{
孙虹霞, 周强, 唐文成, 舒迎花, 张古忍* \\ 中山大学有害生物控制与资源利用国家重点实验室, 昆虫学研究所, 广州 510275 \\ *联系人, E-mail: zhanggr@mail.sysu.edu.cn
}

2008-02-25 收稿, 2008-06-02 接受

国家重点基础研究发展计划(批准号: 2006CB102001)、国家自然科学基金(批准号: 30771458)、教育部博士点专项基金(批准号: 20070558029) 和广东省基础研究团队基金(批准号: E039254)资助项目

摘要 食物中的 $\mathrm{Ni}^{2+}$ 可在斜纹夜蛾 Spodoptera litura Fabricius 幼虫中肠细胞中积累, 并能诱导 中肠细胞中解毒蛋白金属硫蛋白 (metallothionein, MT)的表达. 本文研究了食物中不同剂量 $\mathrm{Ni}^{2+}$ 对 S. litura 5 龄和 6 龄幼虫中肠细胞解毒酶羧酸酯酶 (carboxylesterase, CarE)和谷胱甘肽转 移酶(glutathione S-transferase, GST)活性的影响. 结果表明, S. litura 幼虫连续 3 代取食含不同 剂量 $\mathrm{Ni}^{2+}$ 的食物后, 5 龄幼虫中肠细胞内的 CarE 活性均在低剂量 $\mathrm{Ni}^{2+}(\leq 5 \mathrm{mg} / \mathrm{kg})$ 胁迫下低于 对照, 在高剂量 $\mathrm{Ni}^{2+}(\geq 10 \mathrm{mg} / \mathrm{kg}$ )胁迫下高于对照. 第 1 代 6 龄幼虫中肠细胞内的 CarE 活性也 表现为低剂量 $\mathrm{Ni}^{2+}$ 胁迫抑制而高剂量 $\mathrm{Ni}^{2+}$ 胁迫增加的趋势, 但第 2 和第 3 代 6 龄幼虫中肠细 胞内的 CarE 活性均低于对照. 连续 3 代受不同剂量 $\mathrm{Ni}^{2+}$ 胁迫的 5 龄和 6 龄幼虫中肠细胞的 GST 活性均高于对照, 并随饲料中 $\mathrm{Ni}^{2+}$ 剂量 $(1 \sim 20 \mathrm{mg} / \mathrm{kg}$ )的增加而增加.

关键词

$\mathrm{Ni}^{2+}$

斜纹夜蛾

中肠

羧酸酯酶

谷胱甘肽转移酶
生物体对异源物的解毒在其适应污染环境的过 程中起着重要的作用 ${ }^{[1]}$. 其中, 解毒酶不仅能迅速对 外界环境的变化做出响应, 反映污染物的存在及毒 性, 还可在种群和生态系统水平上预测可能产生的 毒理效应 ${ }^{[2,3]}$.

羧酸酯酶(carboxylesterase, CarE)和谷胱甘肽转 移酶(gulathione-S-transferase, GSTs)分别是生物体中 重要的 | 相和 || 相解毒酶, | 相解毒酶的产物可与内 源的还原型谷胱甘肽 $(\mathrm{GSH})$ 结合, 从而保护体内的蛋 白质和核酸免受损害 ${ }^{[4.5]}$. 此外, 由于分布的广泛性 及在活性氧(reactive oxygen species, ROS)和自由基 代谢中的重要性, 两者还常作为环境污染的指示 物 ${ }^{[6,7]}$. 研究表明, 水生生物和陆生生物体内的 CarE 和 GST 活性在杀虫剂、重金属或矿物质等多种污染 环境下均受到影响 ${ }^{[8 \sim 18]}$; 然而, 对昆虫体内CarE和 GST 的研究多集中于其在杀虫剂污染环境中解毒作用; 在重金属胁迫下, CarE 和 GSTs 活性的变化只在鞘翅目 的Pterosthicus oblongopunctatus ${ }^{[19]}$ 和Poecilus cupreus (L.) ${ }^{[201}$ 以及膜翅目的多栉蚁Formica polyctena
(Först) ${ }^{[21]}$ 中有所报道, 而其在鳞翅目中的变化规律及 与重金属污染程度的关联如何还未见报道.

解毒酶常与金属硫蛋白 (metallothionein, MT)协 同作用以抵御重金属胁迫中的氧化压力. 据 Mosleh 等人 ${ }^{[13,22]}$ 的报道, 污染环境中生物体内MT的表达常 与抗氧化酶如 GSTs 活性的增加相关. 同时, GSTs 所 催化的谷胱甘肽 (glutathione, GSH)也具有转移过量 重金属离子至MT的作用 ${ }^{[23]}$. 我们的前期研究表明, 重金属 $\mathrm{Ni}^{2+}$ 可在鳞翅目昆虫斜纹夜蛾 Spodoptera litura Fabricius幼虫中肠细胞中积累 ${ }^{[24]}$, 并能诱导中 肠细胞中 MT 的表达, 其表达量随中肠细胞中 $\mathrm{Ni}^{2+}$ 量的增加而增加 ${ }^{[24]}$. 然而, 受 $\mathrm{Ni}^{2+}$ 胁迫的S. litura幼 虫中肠细胞中的解毒酶 CarE 和 GST 活性的变化规律 如何? 酶活性与 MT 表达量是否存在一定的关联? 基于这些问题, 本文研究了连续 3 代受不同剂量 $\mathrm{Ni}^{2+}$ 胁迫的 5 龄和 6 龄末 S. litura 幼虫中肠细胞 CarE 和 GST 活性的变化规律, 并探讨了酶活性与 MT 变化 趋势的异同, 以期全面了解 S. litura 幼虫对 $\mathrm{Ni}^{2+}$ 的解 毒机制. 


\section{1 材料和方法}

(i ) 斜纹夜蛾幼虫的饲养与 $\mathrm{Ni}^{2+}$ 胁迫. 斜纹夜 蛾系中山大学昆虫学研究所养虫室人工饲料饲养 ${ }^{[25]}$. 在人工饲料中添加不同剂量的 $\mathrm{Ni}^{2+}$, 并以此连续 3 代饲喂斜纹夜蛾幼虫. 以第 1 代幼虫化蛹后, 羽化成 虫所产的卵作为第 2 代虫源, 第 3 代依此类推, 每天 更换饲料并连续胁迫 3 代. 根据Garrido等人 ${ }^{[26]}$ 的报 道, 当蔬菜中的 $\mathrm{Ni}^{2+}$ 含量达到 $10 \sim 30 \mathrm{mg} / \mathrm{kg}$ 时, 就会 对蔬菜产生毒害作用. 鉴于此, 将 $\mathrm{NiCl}_{2}$ 添加在人工 饲料中, 使 $\mathrm{Ni}^{2+}$ 剂量分别为 $1,5,10,20$ 和 $40 \mathrm{mg} / \mathrm{kg}$. 以不添加 $\mathrm{NiCl}_{2}$ 饲料饲养的斜纹夜蛾为对照. 所有的 昆虫均在 $(26 \pm 1)^{\circ} \mathrm{C}, 16 \mathrm{~L}: 8 \mathrm{D}, \mathrm{RH}(75 \pm 5) \%$ 条件下饲 养.

(ii ) 试剂和仪器. 实验所需 $\alpha$-醋酸萗酚, SDS, 监牢蓝 B，DCNB (1-氯-3,4-二氯硝基苯), GSH 和 EDTA 均购于广州威加公司; 毒扁豆碱购于 Fluka 公 司; 其他试剂均购于广州化学试剂一厂. 紫外可见分 光光度计为 Bio-Rad 系列.

(iii) CarE 活性的测定. 收集长势均一的 5 和 6 龄末 S. litura 幼虫各 10 头, 冰上麻醉和酒精棉球消毒 虫体后, 灭菌锡纸上快速解剖取中肠. 将中肠放入预 冷的 $0.04 \mathrm{~mol} / \mathrm{L} \mathrm{PB}(\mathrm{pH} 7.0)$ 中匀浆后, $4^{\circ} \mathrm{C} 6000 \times \mathrm{g}$ 离 心 $15 \mathrm{~min}$, 取上清液, $0.04 \mathrm{~mol} / \mathrm{L} \mathrm{PB}$ 稀释 20 倍后作为 酶原. 依据Grant等人 ${ }^{[27]}$ 和慕立义 ${ }^{[28]}$ 的方法测定 CarE 活性, 并稍作修改. 反应体系包括 $0.3 \mathrm{~mL}$ 酶原、 $5 \mathrm{~mL}$ 底物 $\left(0.03 \mathrm{~mol} / \mathrm{L} \alpha\right.$-醋酸萗酚 $1 \mathrm{~mL}, 10^{-4} \mathrm{~mol} / \mathrm{L}$ 毒扁豆碱 $1 \mathrm{~mL}, \mathrm{~PB} 98 \mathrm{~mL}, \mathrm{pH}$ 7.0)与 $0.7 \mathrm{~mL} \mathrm{~PB}, 37^{\circ} \mathrm{C}$ 水浴反应 $30 \mathrm{~min}$ 后加入 $1 \mathrm{~mL}$ 显色剂 $(5 \% \mathrm{SDS}$ 和 $1 \%$ 监牢蓝 $\mathrm{B}$ 水溶液 $(2: 5$, 体积比) $)$ 于 $25^{\circ} \mathrm{C}$ 反应 $30 \mathrm{~min}$, 然后于 $600 \mathrm{~nm}$ 处测 $A$ 值. 根据 $\alpha$-萗酚标准曲线求出 酶促生成的量, 酶原中蛋白质经Bradford法 ${ }^{[29]}$ 测定 后, 酶活力表示为 $\mathrm{mmol} \cdot \mathrm{mg}^{-1}$ 蛋白 $\cdot 30 \mathrm{~min}^{-1}$. 每处 理重复 3 次.

(iv) GST 活性的测定. 以上述方法取中肠, 0.1 $\mathrm{mol} / \mathrm{L} \mathrm{PB}(\mathrm{pH} 7.0)$ 匀浆后, $4^{\circ} \mathrm{C} 6000 \times \mathrm{g}$ 离心 $15 \mathrm{~min}$, 取上清液, $0.1 \mathrm{~mol} / \mathrm{L}$ PB 稀释 20 倍, 以此为酶原, 并 依照Habig等人 ${ }^{[30]}$ 的方法测定. 以DCNB为底物, 在 酶的催化下, DCNB 与 GSH 合成的 GSH-S-芳基复合 物在 $340 \mathrm{~nm}$ 处有最大吸收峰. 反应体系为 $3 \mathrm{~mL}$, 其 中包括 $1 \mathrm{mmol} / \mathrm{L} \mathrm{CDNB}, 1 \mathrm{mmol} / \mathrm{L} \mathrm{GSH}, 0.1 \mathrm{~mol} / \mathrm{L}$ $\mathrm{PB}(\mathrm{pH}$ 6.5), $1 \mathrm{mmol} / \mathrm{L}$ EDTA 和 $10 \mu \mathrm{L}$ 酶原; 空白为 未加酶原的反应体系。同时，酶原中蛋白质经
Bradford 法(1976)测定后, 计算出 GST 的比活力 $A \cdot \mathrm{min}^{-1} \cdot \mathrm{mg}^{-1}$ 蛋白. 每处理重复 3 次.

(v) 数据分析. 连续 3 个世代 5 龄和 6 龄幼虫 中肠的 CarE 和 GST 活性经 $\lg (x+1)$ 转换后, 进行方差 分析, 并进行多重比较, 以阐明对照与处理间以及各 个处理间差异的显著性; 同时采用两因素方差分析 检验了饲料中不同的 $\mathrm{Ni}^{2+}$ 剂量和胁迫世代数双因素 对CarE和GST活性的影响 ${ }^{[31]}$.

\section{2 结果和分析}

\subsection{5 龄和 6 龄幼虫中肠的 CarE 活性}

取食含不同剂量 $\mathrm{Ni}^{2+}$ 的人工饲料后, 连续 3 代 5 龄和 6 龄幼虫中肠细胞内的 CarE 活性均受到影响(图 1 和 2). 低剂量 $\mathrm{Ni}^{2+}(\leq 5 \mathrm{mg} / \mathrm{kg})$ 胁迫下, 第 2 代 6 龄幼 虫和第 3 代 5 龄和 6 龄幼虫中肠的 CarE 活性均显著低 于对照; 同时, 受 $5 \mathrm{mg} / \mathrm{kg} \mathrm{Ni}^{2+}$ 胁迫的第 1 代 6 龄幼虫 和第 2 代 5 龄幼虫中肠的 CarE 活性也显著低于对照.

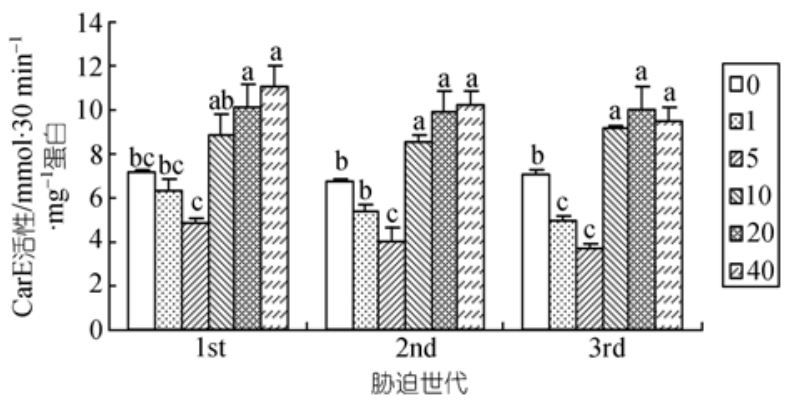

图 1 斜纹夜蛾连续 3 代幼虫取食含不同 $\mathrm{Ni}^{2+}$ 浓度的人工 饲料后 5 龄幼虫中肠内的 CarE 活性

不同小写字母表示幼虫中肠内的 CarE 活性在同一世代不同剂量 $\mathrm{Ni}^{2+}$ 处理中存在显著差异. $\mathrm{Ni}^{2+}$ 浓度单位: $\mathrm{mg} / \mathrm{kg}$

高剂量 $\mathrm{Ni}^{2+}(\geq 10 \mathrm{mg} / \mathrm{kg})$ 胁迫下, 第 2 及 3 代 5 龄幼虫中肠的 CarE 活性显著高于对照; 同时, 受 20 和 $40 \mathrm{mg} / \mathrm{kg} \mathrm{Ni}^{2+}$ 胁迫的第 1 代 5 龄和 6 龄幼虫中肠的 CarE 活性也显著高于对照. 然而, 只有受高剂量 $\mathrm{Ni}^{2+}$ 胁迫的第 3 代 6 龄幼虫中肠的 CarE 活性显著低于对 照, 第 2 代 6 龄幼虫中肠的 CarE 活性均与对照不存 在显著差异.

此外, 除受 $1 \mathrm{mg} / \mathrm{kg} \mathrm{Ni}^{2+}$ 胁迫的第 2 代 6 龄幼虫 外, 低剂量 $\mathrm{Ni}^{2+}$ 胁迫 5 龄和 6 龄幼虫中肠的 CarE 活 性均显著低于高剂量 $\mathrm{Ni}^{2+}$ 胁迫下的活性.

表 1 列出了饲料中 $\mathrm{Ni}^{2+}$ 剂量和胁迫世代数双因素 对斜纹夜蛾 5 龄和 6 龄幼虫中肠 CarE 活性的交互影响. 
结果表明, 饲料中不同剂量的 $\mathrm{Ni}^{2+}$ 浓度对 5 龄和 6 龄 幼虫中肠的 CarE 活性均产生了显著的影响, 但胁迫世 代数只对 6 龄幼虫中肠的 CarE 活性产生显著的影响. 同时, 饲料中 $\mathrm{Ni}^{2+}$ 剂量和胁迫世代数双因素也只对 6 龄幼虫中肠的 CarE 活性产生了显著的交互影响.

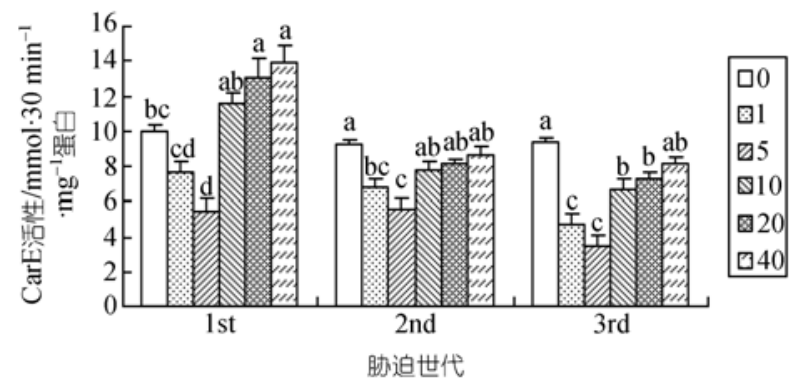

图 2 斜纹夜蛾连续 3 代幼虫取食含不同 $\mathrm{Ni}^{2+}$ 浓度的人工 饲料后 6 龄幼虫中肠内的 CarE 活性

不同小写字母表示幼虫中肠内的 CarE 活性在同一世代不同剂量 $\mathrm{Ni}^{2+}$ 处理中存在显著差异. $\mathrm{Ni}^{2+}$ 浓度单位: $\mathrm{mg} / \mathrm{kg}$

表 1 饲料中 $\mathrm{Ni}^{2+}$ 剂量和胁迫世代数对斜纹夜蛾 5 龄和 6 龄幼虫中肠 CarE 活性的交互影响

\begin{tabular}{clcrrr}
\hline 幼虫龄期 & \multicolumn{1}{c}{ 参数 } & 自由度 & \multicolumn{1}{c}{ 均方 } & \multicolumn{1}{c}{$F$} & \multicolumn{1}{c}{$P$} \\
\hline 5 5 龄 & 饲料中 $\mathrm{Ni}^{2+}$ 剂量 & 5 & 55.015 & 47.38 & $<0.0001$ \\
& 胁迫世代数 & 2 & 2.418 & 2.08 & 0.1393 \\
& 剂量 $\times$ 世代数 & 10 & 0.479 & 0.41 & 0.9315 \\
\hline 6 龄 & 饲料中 $\mathrm{Ni}^{2+}$ 剂量 & 5 & 39.916 & 35.27 & $<0.0001$ \\
& 胁迫世代数 & 2 & 64.035 & 56.59 & $<0.0001$ \\
& 剂量 $\times$ 世代数 & 10 & 5.415 & 4.79 & 0.0002 \\
\hline
\end{tabular}

\subsection{5 龄和 6 龄幼虫中肠的 GST 活性}

连续 3 代受不同剂量 $\mathrm{Ni}^{2+}$ 胁迫的 5 龄和 6 龄幼虫 中肠的 GST 活性的变化如图 3 和 4 所示. 除受 $1 \mathrm{mg} / \mathrm{kg}$ $\mathrm{Ni}^{2+}$ 胁迫的第 2 代 5 龄幼虫外, 连续 3 代受不同剂量 $\mathrm{Ni}^{2+}$ 胁迫的 5 和 6 龄幼虫中肠的 GST 活性均显著高于对照.

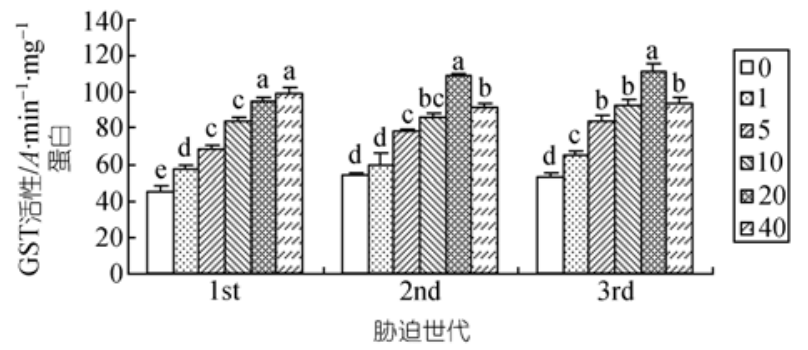

图 3 斜纹夜蛾连续 3 代幼虫取食含不同 $\mathrm{Ni}^{2+}$ 浓度的人工 饲料后 5 龄幼虫中肠内的 GST 活性

不同小写字母表示幼虫中肠内的 GST 活性在同一世代不同剂量 $\mathrm{Ni}^{2+}$ 处理中存在显著差异. $\mathrm{Ni}^{2+}$ 浓度单位: $\mathrm{mg} / \mathrm{kg}$

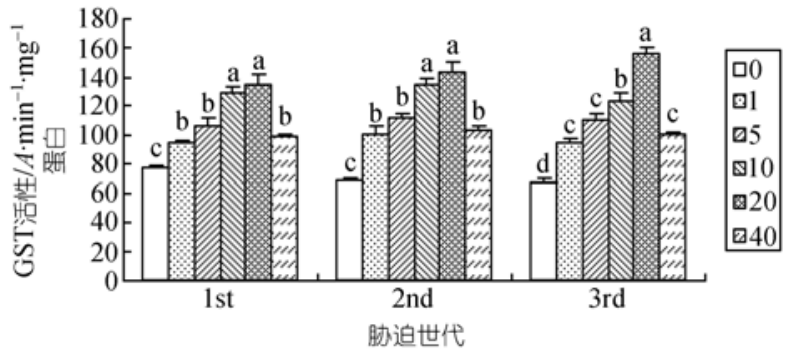

图 4 斜纹夜蛾连续 3 代幼虫取食含不同 $\mathrm{Ni}^{2+}$ 浓度的人工 饲料后 6 龄幼虫中肠内的 GST 活性

不同小写字母表示幼虫中肠内的 GST 活性在同一世代不同剂量 $\mathrm{Ni}^{2+}$ 处理中存在显著差异. $\mathrm{Ni}^{2+}$ 浓度单位: $\mathrm{mg} / \mathrm{kg}$

在 $1 \mathrm{mg} / \mathrm{kg} \mathrm{Ni}^{2+}$ 胁迫下, 5 龄幼虫中肠的 GST 活性 显著低于受 $5 \mathrm{mg} / \mathrm{kg} \mathrm{Ni}{ }^{2+}$ 胁迫幼虫的活性; 且 5 龄和 6 龄幼虫中肠的 GST 活性均显著低于受 10 和 $20 \mathrm{mg} / \mathrm{kg}$ $\mathrm{Ni}^{2+}$ 胁迫幼虫的活性. 在 $5 \mathrm{mg} / \mathrm{kg} \mathrm{Ni}$ 胁迫下, 5 龄幼虫 中肠的 GST 活性显著低于受 $20 \mathrm{mg} / \mathrm{kg} \mathrm{Ni}^{2+}$ 胁迫幼虫的 活性, 而 6 龄幼虫的活性则同时显著低于受 10 和 20 $\mathrm{mg} / \mathrm{kg} \mathrm{Ni}{ }^{2+}$ 胁迫幼虫的活性. 再者, 受 $40 \mathrm{mg} / \mathrm{kg} \mathrm{Ni}^{2+}$ 胁 迫的 5 龄幼虫中肠的 GST 活性显著高于受 1 和 $5 \mathrm{mg} / \mathrm{kg}$ $\mathrm{Ni}^{2+}$ 胁迫的 5 龄幼虫(除第 3 代受 $5 \mathrm{mg} / \mathrm{kg} \mathrm{Ni}^{2+}$ 胁迫幼虫 外)的活性; 6 龄幼虫中肠的 GST 活性则显著低于受 10 和 $20 \mathrm{mg} / \mathrm{kg} \mathrm{Ni}^{2+}$ 胁迫的 6 龄幼虫的活性.

类似的, 饲料中不同剂量的 $\mathrm{Ni}^{2+}$ 浓度也对 5 龄和 6 龄幼虫中肠的 GST 活性均产生了显著的影响. 不同 的是, 胁迫世代数只对 5 龄幼虫中肠的 GST 活性产 生了显著的影响. 同时, 饲料中 $\mathrm{Ni}^{2+}$ 剂量和胁迫世代 数双因素也只对 5 龄幼虫中肠的 GST 活性产生了显 著的交互影响(表 2).

表 2 饲料中 $\mathrm{Ni}^{2+}$ 剂量和胁迫世代数对斜纹夜蛾 5 龄和 6 龄幼虫中肠 GST 活性的交互影响

\begin{tabular}{clrrrr}
\hline 幼虫龄期 & \multicolumn{1}{c}{ 参数 } & 自由度 & \multicolumn{1}{c}{ 均方 } & \multicolumn{1}{c}{$F$} & \multicolumn{1}{c}{$P$} \\
\hline 5 龄 & 饲料中 $\mathrm{Ni}^{2+}$ 剂量 & 5 & 3846.951 & 142.57 & $<0.0001$ \\
& 胁迫世代数 & 2 & 327.794 & 12.15 & $<0.0001$ \\
& 剂量 $\times$ 世代数 & 10 & 64.674 & 2.40 & 0.0268 \\
\hline 6 龄 & 饲料中 $\mathrm{Ni}^{2+}$ 剂量 & 5 & 5980.952 & 110.70 & $<0.0001$ \\
& 胁迫世代数 & 2 & 59.001 & 1.09 & 0.3464 \\
& 剂量 $\times$ 世代数 & 10 & 103.339 & 1.91 & 0.0757 \\
\hline
\end{tabular}

\section{3 讨论}

连续 3 代取食含不同剂量 $\mathrm{Ni}^{2+}$ 食物的 5 龄和 6 龄 幼虫中肠细胞 CarE 和 GST 活性都受到了影响. 其中, 第 2 和第 3 代各处理 S. litura 6 龄幼虫和受低剂量 
$\mathrm{Ni}^{2+}(\leq 5 \mathrm{mg} / \mathrm{kg})$ 胁迫的连续 3 代 5 龄幼虫中肠内的 CarE 活性均受到抑制; 但高剂量 $\mathrm{Ni}^{2+}(\geq 10 \mathrm{mg} / \mathrm{kg})$ 胁 迫则促进 5 龄幼虫和第一代 6 龄幼虫的酶活性. 此变 化趋势与受杀虫剂胁迫的水生生物以及受重金属胁 迫的昆虫体内的 CarE 的变化趋势都有所不同.

水生生物体内的 CarE 活性在有机磷杀虫剂污染 的环境中均受到抑制. 然而, 从污染地带采集的 $P$. oblongopunctatus ${ }^{[19]}$ 和蜘蛛 ${ }^{[32,33]}$ 体内的CarE活性均高 于对照虫体中的活性; 同时, 受不同剂量 $\mathrm{Cd}$ 胁迫的 各发育阶段 Pardosa cupreus 虫体内的 CarE 活性也显 著高于对照虫体中的活性 ${ }^{[20]}$. 不同的是, $\mathrm{Zn}$ 对 $P$. cupreus 体内 CarE 活性的影响则不存在此规律, 受低 剂量 Zn 胁迫幼虫内的 CarE 活性均与对照不存在显 著差异, 高剂量 $\mathrm{Zn}$ 的胁迫则显著降低幼虫体内酶的 活性 ${ }^{[20]}$. 因此, 生物体内的CarE活性与污染物的类 型和剂量、物种的特异性及其发育阶段相关联.

连续 3 代 5 龄和 6 龄 S. litura 幼虫中肠的 GST 活性随饲料中 $\mathrm{Ni}^{2+}$ 剂量 $(1 \sim 20 \mathrm{mg} / \mathrm{kg})$ 的增加而增加. 类似的研究表明, 受重金属 $\mathrm{Pb}$ 和 $\mathrm{Zn}$ 胁迫的狼蛛 $P$. lugubris ${ }^{[33]}$ 和受 $\mathrm{Se}$ 和 $\mathrm{Cu}$ 胁迫的鱼类Leuciscus alburnoides 体内的 GST 活性也与胁迫重金属的剂量正 相关 ${ }^{[34]}$. 同时, 从污染地带收集的蜘蛛Agelena labyrinthica ${ }^{[35]}$ 和F. polyctena $a^{[21]}$ 体内的GST活性也显 著提高.

然而, 在最高剂量 $\mathrm{Ni}^{2+}(40 \mathrm{mg} / \mathrm{kg})$ 胁迫下, 5 龄和 6 龄幼虫中肠的 GST 活性虽高于对照, 但低于受 10 和 20 $\mathrm{mg} / \mathrm{kg} \mathrm{Ni}^{2+}$ 胁迫虫体的 GST 活性, 此现象可能与生物体 对 GSH 的利用有关. GSH 在保护细胞免受毒性物质的 危害中起重要作用 ${ }^{[36]}$, 其活性基团一 $\mathrm{SH}$ 可与过量的重 金属结合, 从而减轻或消除重金属的危害作用, 但同时 也扰乱了GSH相关酶如GST的活性 ${ }^{[37]}$. 受 $40 \mathrm{mg} / \mathrm{kg}$ $\mathrm{Ni}^{2+}$ 胁迫 S. litura 幼虫中肠的 GST 活性的变化可能是为 了相对增加 GSH 的含量, 以消除过量 $\mathrm{Ni}^{2+}$ 的危害.

需要指出的是, 虽然多数文献都报道了 GST 可 迅速地对外界环境的变化起反应, 但 GST 的专一性 较低 ${ }^{[38]}$. Awasthi等人 ${ }^{[39]}$ 的研究表明, Cu对GSTs家族 不同异构酶的活性会产生不同的影响. 而 $\mathrm{Ni}^{2+}$ 是否会 对 S. litura 幼虫不同 GSTs 产生不同的影响还有待于
进一步的研究. 此外, GSH 相关酶如 GST 和 GR (glutathione reductase)和 GPX (glutathione peroxidase) 的活性常相互协调, 以保证体内 GSH含量的平衡 ${ }^{[40]}$; 如受 $\mathrm{Cu}$ 胁迫的正颤蚓 Tubifex tubifex (Müller)体内的 GST活性的变化趋势就和GR活性的趋势相反 ${ }^{[41]}$. 因 此, $\mathrm{Ni}^{2+}$ 胁迫对 S. litura 幼虫体内的 GSH 相关酶活性 的影响也有待于深入探讨.

S. litura 幼虫中肠细胞中的 MT 的表达量也随食 物中 $\mathrm{Ni}^{2+}$ 剂量的增加而增加, 与 $\mathrm{GST}$ 的变化趋势类似; 两者可能存在协同作用, 以共同抵御重金属 $\mathrm{Ni}^{2+}$ 的危 害作用. 通常, MT 的反应灵敏性较酶活性低, 如硬骨 鱼 Channa punctatus (Bloch)体内的 GST 可迅速对 $\mathrm{Cu}$ 的胁迫起反应, 且活性随 $\mathrm{Cu}$ 胁迫时间的延长而增加; 但在 $\mathrm{Cu}$ 胁迫的第 60 天MT才被诱导表达 ${ }^{[8]}$. 然而, 若 $\mathrm{MT}$ 被 $\mathrm{Cu}$ 饱和后, GST 的活性常会有所下降, MT 在生 物体对重金属的解毒过程中发挥更重要的作用 ${ }^{[41]}$. 对 S. litura 幼虫而言, 其中肠的 MT 能否被饱和, 而在其 被饱和后 GST 的变化趋势如何等问题还有待于进一 步的验证.

5 龄和 6 龄幼虫中肠的 CarE 的变化趋势有所不 同, 不同发育阶段幼虫的生理特性的变化可能是此 差异的主要原因, 同时, 发育阶段特异性的酶活性变 化在 $P$. cupreus等昆虫中也有所报道 ${ }^{[20]}$. 再者, 生物 体对污染物的解毒是能量消耗的过程, 有限的能量 储存决定生物体会将能量优先投入到具高敏感性和 高效率的生理反应中 ${ }^{[19]}$; 虽然GST和CarE的专一性 均较低, 但 GST 在重金属解毒过程中的稳定性和敏 感性较均CarE高 ${ }^{[35]}$. 因此, 斜纹夜蛾幼虫可能将较 多的能量转至 GST 和 MT 的增加上, 从而迅速和稳定 地防御重金属 $\mathrm{Ni}^{2+}$ 的危害作用; 而有限的能量存储虽 可提高受高剂量 $\mathrm{Ni}^{2+}$ 胁迫 5 龄幼虫中肠的 $\mathrm{CarE}$ 活性, 但最终降低了 6 龄幼虫中肠的 CarE 活性.

研究表明, 连续 3 代 $\mathrm{Ni}^{2+}$ 的胁迫影响了 5 龄和 6 龄幼虫中肠解毒酶 CarE 和 GST 的活性. GST 可能和 MT 协同作用以抵御重金属的危害作用. 然而, 斜纹 夜蛾幼虫体内是否存在其他的解毒作用, 而幼虫对 过量重金属解毒的具体机制还有待于研究和探讨.

\section{参考文献}

Jokanovic M. Biotransformation of organophosphorus compounds. Toxicology, 2001, 166: 139-160[DOI]

Scott-Fordsmand J J, Weeks J M. Biomarkers in earthworms. Rev Environ Contam Toxicol, 2000, 165: 117-159

Nicholson S, Lam P K S. Pollution monitoring in Southeast Asia using biomarker in the mytilid mussel Perna viridis (Mytilidae: Bival- 
via). Environ Int, 2005, 31: 121-132[DOI]

4 Gadagbui B K, James M O. Activities of affinity-isolated glutathione S-transferase (GST) from channel catfish whole intestine. Aquatic Toxicol, 2000, 19: 27-37[DOI]

5 Norppa H. Genetic susceptibility, biomarker responses and cancer. Mutat Res, 2003, 544: 339-348 [DOI]

6 Company R, Serafim A, Bebianno M J, et al. Effect of cadmium, copper and mercury on antioxidant enzyme activities lipid peroxidation in the gills of the hydrothermal vent mussel Bathymodiolus azoricus. Mar Environ Res, 2004, 58: 377 - 381 [DOI]

7 Rajalakshmi S, Mohandas A. Copper-induced changes in tissue enzyme activity in a freshwater mussel. Ecotoxicol Environ Saf, 2005, 62: $140-143$ [DOI]

8 Ahmad A, Hamid T, Fatima M, et al. Induction of hepatic antioxidants in freshwater catfish (Channa punctatus Bloch) is a biomarker of paper mill effluent exposure. Biochim Biophys Acta, 2000, 1523: 37-48

9 Galloway T S, Millward N, Browne M A, et al. Rapid assessment of organophosphorous/carbamate exposure in the bivalve mollusc Mytilus edulis using combined esterase activities as biomarkers. Aquat Toxicol, 2002, 61: 169-180[DOI]

10 Bonacci S, Browne M A, Dissanayake A, et al. Esterase activities in the bivalve mollusc Adamussium colbecki as a biomarker for pollution monitoring in the Antarctic marine environment. Mar Pollut Bull, 2004, 49: 445-455[DOI]

11 Geracitano L A, Bocchetti R, Monserrat J M, et al. Oxidative stress responses in two populations of Laeonereis acuta (Polychaeta, Nereididae) after acute and chronic exposure to copper. Mar Environ Res, 2004, 58: 1-17[DOI]

12 Küster E. Cholin- and carboxylesterase activities in developing zebrafish embryos (Danio rerio) and their potential use for insecticide hazard assessment. Aquat Toxicol, 2005, 75: 76-85[DOI]

13 Mosleh Y Y, Paris-Palacios S, Couderchet M, et al. Metallothionein induction, antioxidative responses, glycogen and growth changes in Tubifex tubifex (Oligochaete) exposed to the fungicide, fenhexamid. Environ Pollut, 2005, 135: 73-82[DOI]

14 Wheeloch C E, Eder K J, Werner I, et al. Individual variability in esterase activity and CYP1A levels in Chinook salmon (Oncorhynchus tshawytscha) exposed to esfenvalerate and chlorpyrifos. Aquat Toxicol, 2005, 74: 172-192 [DOI]

15 Zanette J, Monserrat J M, Bianchini A. Biochemical biomarkers in gills of mangrove oyster Crassostrea rhizophorae from three Brazilian estuaries. Comp Biochem Physiol, Part C, 2006, 143: 187-195

16 Cunha I, Mangas-Ramirez E, Guilhermino L. Effects of copper and cadmium on cholinesterase and glutathione S-transferase activities of two marine gastropods (Monodonta lineate and Nucella lapillus). Comp Biochem Physiol, Part C, 2007, 145: 648-657

17 Ferrari A, Venturino A, de D'Angelo A M P. Effects of carbaryl and azinphos methyl on juvenile rainbow trout (Oncorhynchus mykiss) detoxifying enzymes. Pestic Biochem Physiol, 2007, 88: 134-142[DOI]

18 Vioque-Fernández A, de Almeida E A, López-Barea J. Esterase as pesticide biomarkers in crayfish (Procambarus clarkia, Crustacea): Tissue distribution, sensitivity to model compounds and recovery from inactivation. Comp Biochem Physiol, Part C, 2007, 145: 404-412

19 Stone D, Jepson P, Laskowski R. Trends in detoxification enzymes and heavy metal accumulation in ground beetles (Coleoptera: Carabidae) inhabiting a gradient of pollution. Comp Biochem Physiol, Part C, 2002, 132: 105-112

20 Wilczek G, Kramarz P, Babczyń ska A. Activity of carboxylesterase and glutathione S-transferase in different life-stages of carabid beetle (Poecilus cupreus) exposed to toxic metal concentrations. Comp Biochem Physiol, Part C, 2003, 134: 501-512

21 Migula P, G1 owacka E. Heavy metals as stressing factors in the red wood ants (Formica polyctena) from industrially polluted areas. Fresenius' J Anal Chem, 1996, 354: 653-659

22 Mosleh Y Y, Paris-Palacios S, Couderchet M, et al. Effects of the herbicide isoproturon on metallothionein, growth, and antioxidative defenses in the aquatic worm Tubifex tubifex (Oligochaete, Tubificidae). Ecotoxicology, 2005, 43: 1-13

23 Freedman J H, Ciriolo M R, Peisach J. The role of glutathione in copper metabolism and toxicity. J Biol Chem, 1989, 264: 5598-5605

24 Sun H X, Zhou Q, Tang W C, et al. Metallothionein expression induced by nickel accumulation in the midgut of Spodoptera litura Fabricius larvae exposed to nickel. Chin Sci Bull, 2007, 52: 3227-3232

25 陈其津，李广宏，庞义。饲养 5 种夜蛾科昆虫的一种简易人工饲料. 昆虫知识, 2000, 37(6): 325一 327

26 Garrido S, Campo G M C, Esteller M V, et al. Heavy metals in soil treated with sewage sludge composting, their effects on yield and uptake of broad bean seeds (Vicia faba L.). Water Air Soil Pollut, 2005, 166: 303-319[DOI]

27 Grant D F, Bender D M, Hammock B D. Quantitative kinetic assays for glutathione S-transferase and general esterase in individual mosquitoes using an EIA reader. Insect Biochem, 1989, 19: 741-751[DOI]

28 慕立义. 植物化学保护研究方法. 北京: 中国农业出版社, 1994. 146-163

29 Bradford M M. A rapid and sensitive method for the quantitation of microgrma quantities of protein utilizing the principal of protein-dye binding. Anal Biochem, 1976, 72: 248-254

30 Habig W H, Pabst M J, Jakoby W B. Glutathione S-transferase: The first enzymatic step in mercapturic acid formation. J Biol Chem, 1974, 249: $7130-7139$

31 SAS Institute Inc. SAS/STAT User's Guider. Ver 6, 4th ed. Cary, NC: SAS Institute Inc, 1989. Vol 1 and 2

32 Wilczek G, Babczyń ska A, Migula P, et al. Activity of esterase as biomarkers of metal exposure in spiders from the metal pollution gradient. Polish J Environ Stud, 2003, 6: 765-771

33 Wilczek G, Babczyń ska A, Augustyniak M, et al. Relations between metals ( $\mathrm{Zn}, \mathrm{Pb}, \mathrm{Cd}$ and $\mathrm{Cu})$ and glutathione-dependent detoxifying enzymes in spiders from a heavy metal pollution gradient. Environ Pollut, 2004, 132: 453-461 [DOI]

34 Lopes P A, Pinheiro T, Santos M C, et al. Response of antioxidant enzymes in freshwater fish populations (Leuciscus alburnoides complex) to inorganic pollutants exposure. Sci Total Environ, 2001, 280: 153-163[DOI]

35 Babczyń ska A, Wilczek G, Migula P. Effects of dimethoate on spiders from metal pollution gradient. Sci Total Environ, 2006, 370: 352-359[DO]]

36 Anderson M E, Luo J L. Glutathione therapy: from prodrugs to genes. Semin Liver Dis, 1998, 18: 415-424

37 Diaz-Cruz M S, Mendieta J, Tauler R, et al. Cadmium-binding properties of glutathione: A chemometrical analysis of voltammetric data. J Inorg Biochem, 1997, 66: 29-36[DOI

38 McLoughlin N, Yin D, Maltby L, et al. Evaluation of sensitivity and specificity of two crustacean biochemical biomarkers. Environ Toxicol Chem, 2000, 19: 2085-2091[DOI]

39 Awasthi Y C, Zimniak P, Awasthi S, et al. A new group of glutathione S-transferase with a protective role against lipid peroxidation. In: Vermeulen N P E, Mulder G H, Nieuwenhuyse H, et al, eds. Glutathione S-transferase, Structure, Function and Clinical Implications. London: Taylor and Francis Ltd, 1996. 111-124

40 Łaszczyca P, Augustyniak M, Babczyńska A, et al. Profiles of enzymatic activity in earthworm from zinc, lead and cadmium polluted areas near Olkusz (Poland). Environ Int, 2004, 30: 901-910[DOI]

41 Mosleh Y Y, Paris-Palacios S, Biagianti-Risbourg S. Metallothioneins induction and antioxidative response in aquatic worms Tubifex tubifex (Oligochaete, Tubificidae) exposed to copper. Chemosphere, 2006, 64: 121-128[DOI] 